\title{
Predicting the residual aluminum level in water treatment process
}

\author{
J. Tomperi ${ }^{1}$, M. Pelo ${ }^{2}$, and K. Leiviskä ${ }^{1}$ \\ ${ }^{1}$ University of Oulu, Control Engineering Laboratory, P.O. Box 4300, 90014 University of Oulu, Finland \\ ${ }^{2}$ Finnsugar Ltd, Sokeritehtaantie 20, 02460 Kantvik, Finland \\ Correspondence to: J. Tomperi (jani.tomperi@oulu.fi)
}

Received: 18 May 2012 - Published in Drink. Water Eng. Sci. Discuss.: 27 June 2012

Revised: 10 April 2013 - Accepted: 10 May 2013 - Published: 3 June 2013

\begin{abstract}
In water treatment processes, aluminum salts are widely used as coagulation chemical. High dose of aluminum has been proved to be at least a minor health risk and some evidence points out that aluminum could increase the risk of Alzheimer's disease. Thus it is important to minimize the amount of residual aluminum in drinking water and water used at food industry. In this study, the data of a water treatment plant (WTP) was analyzed and the residual aluminum in drinking water was predicted using Multiple Linear Regression (MLR) and Artificial Neural Network (ANN) models. The purpose was to find out which variables affect the amount of residual aluminum and create simple and reliable prediction models which can be used in an early warning system (EWS). Accuracy of ANN and MLR models were compared. The new nonlinear scaling method based on generalized norms and skewness was used to scale all measurement variables to range $[-2 \ldots+2]$ before data-analysis and modeling. The effect of data pre-processing was studied by comparing prediction results to ones achieved in an earlier study. Results showed that it is possible to predict the baseline level of residual aluminum in drinking water with a simple model. Variables that affected the most the amount of residual aluminum were among others: raw water temperature, raw water $\mathrm{KMnO}_{4}$ and $\mathrm{PAC} / \mathrm{KMnO}_{4}(\mathrm{Poly}$-Aluminum Chloride/Potassium permanganate)-ratio. The accuracies of MLR and ANN models were found to be almost the same. Study also showed that data pre-processing affects to the final prediction result.
\end{abstract}

\section{Introduction}

In water treatment processes surface waters are most commonly treated with chemical coagulation. Aluminum salts are widely used as a coagulant to reduce the organic matter, color and turbidity of raw water. Using aluminum salts in a water treatment process may lead to an increased concentration of aluminum in drinking water if aluminum is overdosed or the water treatment process is dysfunctional. The residual aluminum increases the water turbidity, may have some health effects on consumers and aluminum hydroxide may deposit on the walls of the pipes decreasing flow capacity (Driscoll and Letterman, 1995; WHO, 2008). Reported minor symptoms of the high level of residual aluminum in drinking water are nausea, vomiting, diarrhea, mouth and skin ulcers, rashes and arthritic pain (WHO, 2003). Symptoms are generally mild and short-lived. More serious health effects of aluminum in drinking water have been studied widely and the results are conflicting. A Canadian study of health and aging claims that residual aluminum in drinking water does not increase the risk of Alzheimer's disease (Leakey, 2004). However, several researches that showed relationships between aluminum in drinking water and Alzheimer's disease have been found in George et al. (2010), Mclachlan et al. (1996), WHO (2008).

The total intake level of aluminum from drinking water varies according to the aluminum level in raw water and whether aluminum coagulants are used in a water treatment process. The concentration of aluminum in natural waters can vary significantly depending on various physicochemical and mineralogical factors. The aluminum intake from food and water is unavoidable but only $5 \%$ of the total intake is from drinking water. The major part $\left(5 \mathrm{mg} \mathrm{day}^{-1}\right)$ of 
the total intake comes from food and its additives (WHO, 2003, 2008).

Juntunen et al. (2010) found that the most important parameters that affect the amount of residual aluminum in drinking water were the raw water temperature, $\mathrm{Al} / \mathrm{KMnO}_{4}$ ratio, turbidity and silicate concentration. The treated water $\mathrm{pH}$, the $\mathrm{pH}$ of coagulation and the temperature and turbidity of water were found to have an effect on the amount of residual aluminum in Driscoll and Letterman (1995). WHO (2008) reported that the residual aluminum concentration in drinking water can be minimized by optimizing $\mathrm{pH}$, avoiding excessive dosing of aluminum, good mixing of coagulants, optimum paddle speed in the flocculation process and efficient floc filtration.

Artificial Neural Networks (ANN) has been reported to have many benefits against traditional data modeling methods. Data-driven ANN can capture relationships using the desired input output mapping and physical processes do not have to be known explicitly like when using mechanistic models. In a drinking water treatment process modifications can occur frequently and very often micro-scale interactions are poorly understood. This makes it impossible to develop a useful mechanistic model. Using an ANN model gives the ability to quickly modify process models using full-scale operation data without necessity to understand all micro-scale interactions (Baxter et al., 2001; Maier et al., 2004).

The performance of Multiple Linear Regression (MLR) and Artificial Neural Network models has been compared in several studies (Juntunen et al., 2010; Maier et al., 2004; Bowden et al., 2006; Ibarra-Berastegi et al., 2007; Areerachakul and Sanguansintukul, 2009; Kulkarni and Chellan, 2010) and ANN models have been found to outperform the MLR models in training, testing and validation of different prediction cases. However, the difference was not always significant. The supremacy of ANN models indicates nonlinear relationships in used datasets. In the water treatment and drinking water production, ANN models have been successfully used to among others modeling and predicting chlorine residual in a water distribution system (Bowden et al., 2006), drinking water quality (Baxter et al., 2001), contaminant removal (Shetty et al., 2003), fouling and backwash efficiency in ultrafiltration (Delgrange-Vincent et al., 2000), optimal aluminum doses (Maier et al., 2004) and residual aluminum (Juntunen et al., 2010) in the water treatment process. Baxter et al. (2001) created also a prediction model to provide plant operators with an early warning system (EWS) for raw water quality changes and to improve treatment efficiency.

In this paper the data of a water treatment plant is analyzed and prediction models are created using traditional linear and nonlinear methods. The purpose was to find out which variables affect the amount of residual aluminum in drinking water and to study if it is possible to predict reliably the residual aluminum level using only a few important measurement variables. Information of the reliable simple prediction model could be used for a EWS at the plant, to activate inhibitory actions by process operators or on-line control systems to avoid increased values of residual aluminum. The performance of MLR and ANN models is compared with each other and to earlier prediction results presented in Juntunen et al. (2010). Same modeling methods and the same data source were used to study the effect of data pre-processing (scaling, interpolation, averaging) to modeling accuracy.

\section{Material and methods}

\subsection{The water treatment plant}

The data was collected from the water treatment plant (WTP) of Finnsugar Ltd. in Kirkkonummi, Finland. This chemical treatment plant uses surface water from a lake nearby (Humaljärvi), an artificial lake (Pikkala) or the mixture of these two sources as raw water. Before adding the coagulation chemical the $\mathrm{pH}$ of raw water is adjusted to the optimal value with calcium hydroxide. Aluminum based coagulation chemical PAX-14 (Kemira Kemwater) is used in the coagulation process. The coagulation chemical dose is controlled as a function of raw water $\mathrm{KMnO}_{4}$ (potassium permanganate) value. After the filtration, water $\mathrm{pH}$ is again adjusted to the optimal level for distribution. UV-radiation and sodium hypochlorite are used for disinfection. The process stages of the WTP is shown in Fig. 1.

During the period of data collection, the long-term mean value of residual aluminum in drinking water produced at Finnsugar Ltd. WTP was less than half of the maximum target value of the quality recommendation $\left(0.2 \mathrm{mg} \mathrm{l}^{-1}\right)$ defined in the Health Protection Act of the Finland's Ministry of Social Affairs and Health (FINLEX, 2000). Thus, a high amount of residual aluminum in drinking water is not a serious concern in this water treatment plant. Even so, Finnsugar Ltd. has a great interest to find out which variables have an effect on the amount of residual aluminum and use this information to keep it at minimum level.

\subsection{Dataset}

The quality of the developed model depends highly on the quality of the source dataset. The dataset used in analyses and modeling the water treatment process must be fully representative of the full spectrum of all possible conditions. The temperature of surface water, for instance, varies depending on the season of the year. Therefore the source dataset must encompass at least one full year of measured data (Baxter et al., 2001).

The dataset used in analysis and modeling in this study covered a period of 16 months. The dataset included online process measurement variables, and laboratory measurements of raw water and drinking water. Only the measurements which could be used for the prediction of residual aluminum in drinking water were used, thus the measurements 


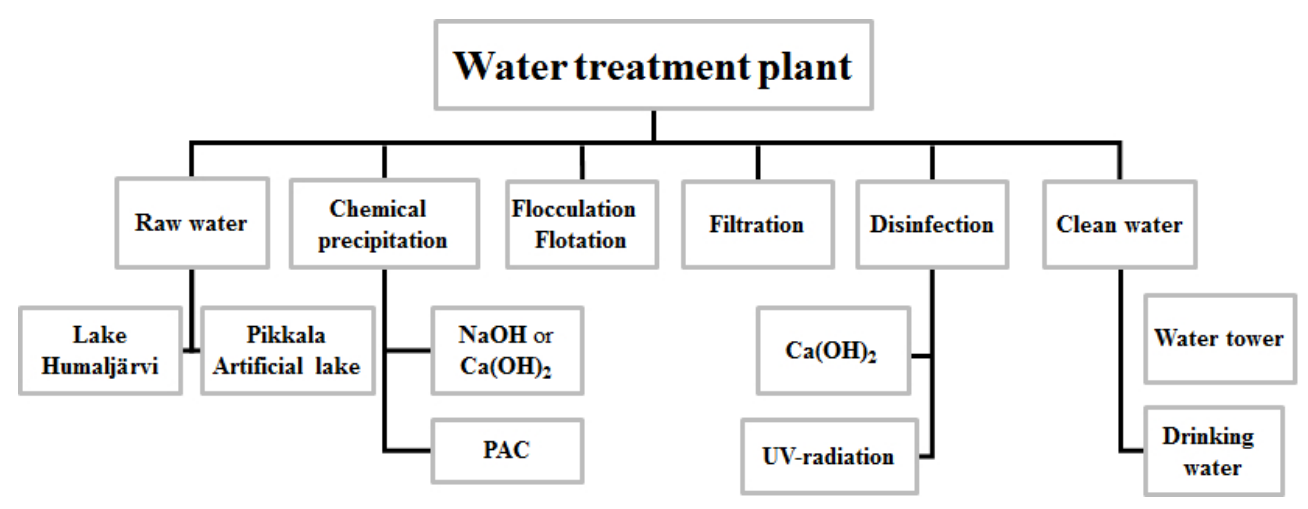

Figure 1. Processing stages of the Finnsugar Ltd. water treatment plant.

of drinking water were not used in modeling the residual aluminum. Measured laboratory variables were $\mathrm{pH}$, potassium permanganate, turbidity, hardness, color, conductivity, smell, chlorine, bacteria and aluminum.

The laboratory measurements of raw and drinking waters were done at least once in every working day. If some measurement results showed anomalous values, new samples were collected and analyzed. For the data analysis, all on-line measurements, which were originally stored at $5 \mathrm{~min}$ intervals, were averaged to one hour data. Laboratory measurement values were combined to the corresponding hour of on-line measurement data. Few evident outliers (values that were not realistic) were manually filtered out and missing data values were added by linear interpolation using inpaint_nans-Matlab function created by D'Errico (2004).

\subsection{Nonlinear scaling}

A dataset which contains several different measurement variables has to be scaled before data analysis to facilitate analysis and avoid incorrect conclusions. The inspection of the raw dataset may not reveal all the noteworthy changes or states. After scaling different variables can easily be used in calculations and the real values of measurements are not revealed. In this work, the dataset was scaled between $[-2 \ldots+2]$ using the new nonlinear scaling method based on generalized norms and skewness.

Nonlinear mapping function has been developed in Juuso (2010) and Juuso (2011) to extract the meanings of variables from measurement signals. These functions are called membership definitions. Membership definitions map the real values of variables to the range of $[-2 \ldots+2]$. Thus, a normal scaling to range $[-1 \ldots+1]$ is combined with handling of warnings and alarms. A trapezoidal membership function which is based on the support and core areas defined by fuzzy set theory is used to define the concept of the feasible range. The support area is defined by the minimum, $\min \left(x_{j}\right)$, and maximum, $\max \left(x_{j}\right)$, of the values of the variable. The value range $x_{j}$ is divided into two parts by the central tendency value $c_{j}$. The core area $\left[\left(c_{l}\right)_{j},\left(c_{h}\right)_{j}\right]$ is limited by the central tendency values of the lower and upper part. The mapping function contains one monotonously increasing function for the values between -2 to 0 and one monotonously increasing function between values 0 to +2 . Membership functions consist of two second order polynomials: one for the negative values and one for the positive values presented in Eq. (1).

$$
\begin{aligned}
& f_{j}^{-}=a_{j}^{-} X_{j}^{2}+b_{j}^{-} X_{j}+c_{j}, X_{j} \in[-2,0), \\
& f_{j}^{+}=a_{j}^{+} X_{j}^{2}+b_{j}^{+} X_{j}+c_{j}, X_{j} \in[0,2] .
\end{aligned}
$$

Because the scaling idea is based on the membership functions of fuzzy set systems these values are called linguistic values. The coefficients of the polynomials are defined by the corner points

$\left.\left.\left\{\min \left(x_{j}\right),-2\right),\left(\left(c_{l}\right)_{j},-1\right), c_{j}, 0\right),\left(\left(c_{h}\right)_{j}, 1\right), \max \left(x_{j}\right), 2\right\}$.

The detailed description of this new nonlinear scaling method is presented in Juuso $(2010,2011)$.

\subsection{Variable selection}

Variable selection is one of the most important steps in the model development process. The greater number of variables does not necessary mean better prediction results. Some input variables may be correlated, noisy or have no significant relationship with the output variable and thus will not be informative. Selecting non-essential inputs only increases computational complexity, makes the training process more difficult and prediction results worse (Bowden et al., 2006).

In this work, variable selection was done using the stepwise regression, a forward selection method, which adds the best variable to, or deletes the worst variable from a variable subset at each round. Adding and deleting is based on variables statistical significance ( $p$ value) in regression. The forward selection method starts with an initial model and continues until either no further model changes occur over one complete round or a preset number of variable selections and deletions occur. Manually selected variables were also used 
in modeling to test if it is possible to create a good and simple model using only a few important variables. Another goal was to compare the results with ones presented in Juntunen et al. (2010) and see the effect of data pre-processing on modeling accuracy.

\subsection{Multiple Linear Regression}

Multiple Linear Regression can be used to describe a quantitative relationship between several independent variables and a dependent variable as a linear system, to predict future scores on the dependent variable or to test specific hypotheses based on a scientific theory or prior research. A linear equation is fitted to observe independent variables. MLR equation is a weighted linear combination of the independent variables and can be written as presented in Eq. (3) (Areerachakul and Sanguansintukul, 2009; Matlab, 2011; Tranmer and Elliot, 2008; Audone and Giunta, 2008).

$Y=b_{0}+b_{1} X_{1}+b_{2} X_{2}+\ldots+b_{n} X_{n}+e$

where $b_{o}$ is a constant value, $b_{1} \ldots b_{n}$ are regression coefficients, $X_{1} \ldots X_{n}$ independent variables and $e$ is the error.

The major limitations of MLR are that it may not be useful with nonlinear features and that one can only ascertain relationships, but not be sure about underlying causal mechanism.

\subsection{Artificial Neural Network}

An Artificial Neural Network typically consists of at least three layers: an input layer, one or more hidden layers and an output layer. External inputs of the network are received by neurons in the input layer. Inputs are multiplied by interconnection weights and sent forward to the hidden layer where they are summed and processed by a nonlinear transfer function. Each value from the input layer is sent to every neuron in the hidden layer. If the network has more than one hidden layer, data is multiplied by interconnection weights, summed and processed by a transfer function in every layer before it is sent to the output layer. The output of the network is given by the neurons on the output layer (Maier et al., 2004; Dayhoff, 1990).

The multilayer perceptron (MLP) is the most common neural network model. MLP is a feedforward ANN which utilizes a supervised learning technique called backpropagation for training a network. Neural networks are trained by examples using historical data. The three-layer back-propagation network is one of the most used architectures in process modeling. Back-error propagation, or backpropagation, is widely and successfully used in Neural Network paradigms because it is easy to understand. The aim of the training process is to minimize the output error by adjusting the interconnection weights which are set at random values at the beginning of the training. The error is defined to be the difference between the predicted output and measured output. The calculated error is back-propagated to the neural network through each layer and the weights are adjusted to decrease the error. The training process is continued until the error is minimized and the network has learned the data (Baxter et al., 2001; Maier et al., 2004; Delgrange-Vincent et al., 2000; Dayhoff, 1990; Pal and Mitra, 1992; Beale et al., 2010).

The number of hidden layers, the number of neurons, the learning rate and initial weights, for instance, can influence the network training and prediction accuracy (Maier et al., 2004; Bowden et al., 2006). The optimum number of hidden layers and nodes are often found by trial and error. It has been proven that one hidden layer can give sufficient degree of freedom but using more than one hidden layer provides greater flexibility and enables the approximation of complex functions with fewer connection weights (Maier et al., 2004; Delgrange et al., 1998).

In this work the Neural Network consisted of measured variables as inputs, the predicted value of residual aluminum as output and one hidden layer (5 neurons). Resilient backpropagation was used as the training function and the mean squared error (MSE) as the performance function. Hyperbolic tangent sigmoid was used as the transfer function for the hidden layer, and the linear transfer function for the output layer. The configuration was selected similar as used in the study of Juntunen et al. (2010) so that the comparison of prediction results would be easier and more reliable.

Hyperbolic tangent sigmoid is defined as Eqs. (4) and (5).

$f(s)=\frac{1-e^{-2 s}}{1+e^{-2 s}}$,

where

$e=\sum_{i=1}^{n} w_{i} x_{i}+b$

where $w_{i}$ are the weights, $x_{i}$ are the inputs of neurons, $b$ is a bias and $n$ is the number of variables.

Performance of ANN and MLR models can be evaluated for example using Root Mean Square Error (RMSE), Mean Absolute Error (MAE) and coefficient of determination $\left(R^{2}\right)$. MAE can be used to determine whether model predictions are suitable for process control. $R^{2}$ value can be used to compare the relative performance of the models (Baxter et al., 1999).

The coefficient of determination value $R^{2}$ is defined as in Eq. (6), RMSE is defined as in Eq. (7) and MAE is defined as in Eq. (8).

$$
R^{2}=1-\frac{\sum\left(y_{\text {meas }}-y_{\text {pred }}\right)^{2}}{\sum\left(y_{\text {meas }}-\frac{\sum y_{\text {meas }}}{k}\right)^{2}}
$$

where $y_{\text {meas }}$ is a measured value, $y_{\text {pred }}$ is a predicted value and $k$ is the number of values.

$\operatorname{RMSE}=\sqrt{\frac{1}{k} \sum\left(\left(y_{\text {meas }}-y_{\text {pred }}\right)^{2}\right)}$ 


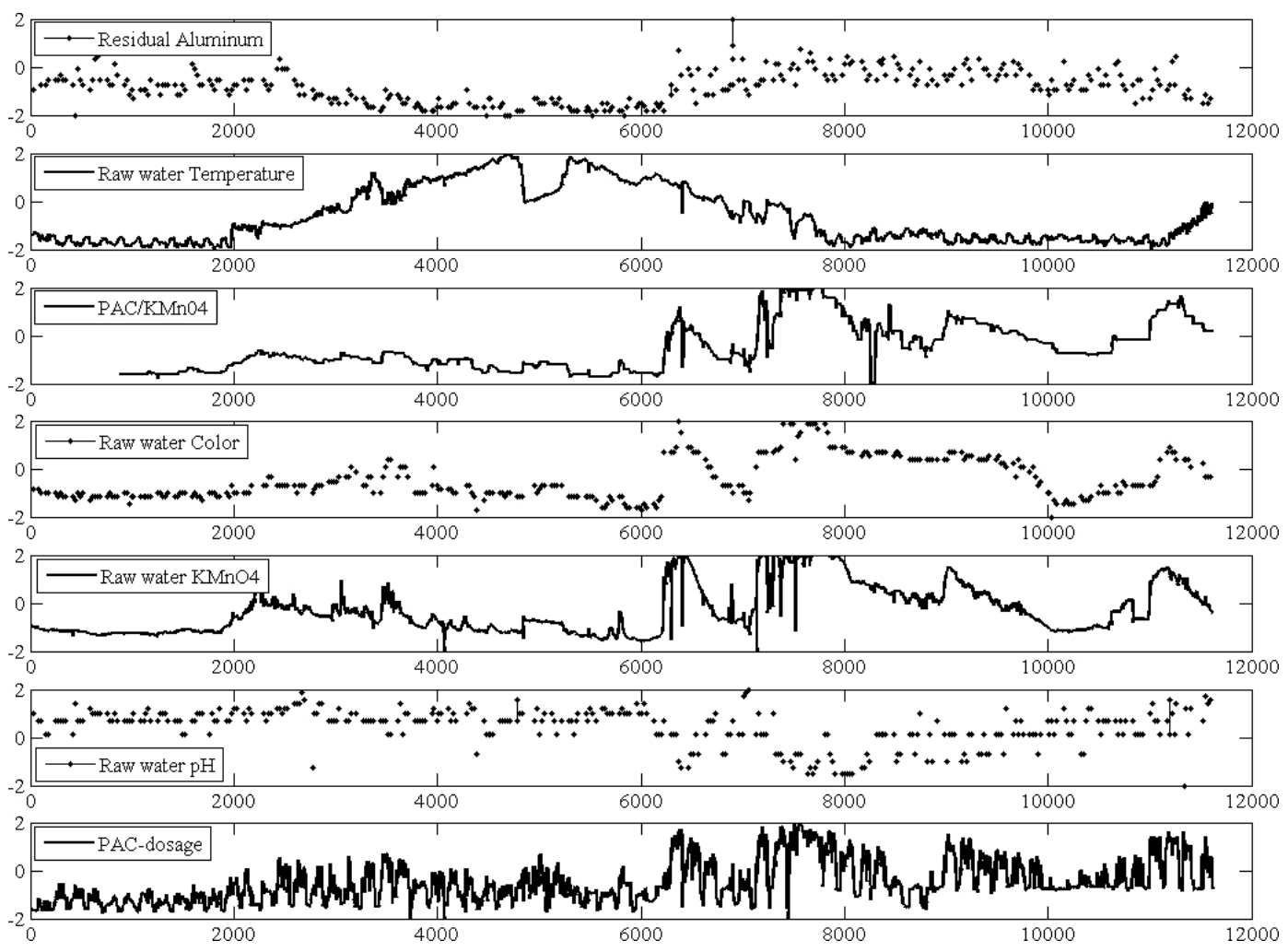

Figure 2. Trend lines of residual aluminum and important measurement variables. From top to down: residual aluminum, raw water temperature, $\mathrm{PAC} / \mathrm{KMnO}_{4}$, raw water color, raw water $\mathrm{KMnO}_{4}$, raw water $\mathrm{pH}$ and $\mathrm{PAC}$-dosage.

where $y_{\text {meas }}$ is a measured value, $y_{\text {pred }}$ is a predicted value and $k$ is the number of values.

MAE $=\frac{1}{k} \sum\left|y_{\text {preds }}-y_{\text {meas }}\right|$

where $y_{\text {meas }}$ is a measured value, $y_{\text {pred }}$ is a predicted value and $k$ is the number of values.

\section{Results and discussion}

\subsection{General study}

The combined on-line and laboratory measurement dataset was studied to find out significant correlations between measured variables and residual aluminum. The highest correlated variables to residual aluminum are shown in Table 1 sections (A), (B), (C) and (D). It can be seen that results of correlation determination varied depending on the order of the nonlinear scaling and data interpolation. Section (A) shows the correlation coefficients of the original, unprocessed, dataset. Only the laboratory measurements of raw water had high correlation to residual aluminum and all online measurements had very low correlation coefficients. In section (B) correlation coefficients are presented when the dataset was first scaled and then interpolated. Good correlations of some on-line measurements were now revealed. The new nonlinear scaling method clearly improved the ability to identify interactions of measurement variables and changes in the specific trend line compared with original data trends. The number of variables with good correlation and values of correlation coefficients decreased if the original data was at first interpolated and then scaled, which can be seen in section (D). This indicates that there are several Not a Number, in other words missing values, in the measurement data of that variable. Section (C) shows the correlation coefficients of residual aluminum from the dataset which consists only of the on-line and laboratory measurement values at the exact time of drinking water sampling. Interpolation did not affect the results of the correlation calculation. Selecting the pre-processing method of the dataset is important because the quality of the data affects the accuracy of prediction models.

It can be seen in Table 1 that certain variables, like raw water temperature, $\mathrm{PAC} / \mathrm{KMnO}_{4}$ (Poly-Aluminum Chloride/Potassium permanganate)-ratio, raw water color, raw water $\mathrm{KMnO}_{4}$ and $\mathrm{pH}$, always have high correlation with residual aluminum. The temperature, $\mathrm{pH}$ and $\mathrm{PAC} / \mathrm{KMnO}_{4}-$ ratio were found to be affecting variables to residual aluminum also in earlier studies of Driscoll and Letterman (1995) and Juntunen et al. (2010).

Trend lines of residual aluminum and the highest correlated variables are shown in Fig. 2. Seasonal changes in raw water temperature are clearly seen at the second subfigure. 
Table 1. Correlation coefficients of the residual aluminum.

\begin{tabular}{|c|c|c|c|}
\hline \multicolumn{2}{|l|}{ (A) Original Dataset } & \multicolumn{2}{|c|}{ (B) Scaled and interpolated dataset } \\
\hline Correlation coefficient & Variable & Correlation coefficient & Variable \\
\hline 0.82 & Raw water color (lab) & -0.61 & Raw water temperature (on-line) \\
\hline 0.81 & Raw water pH (lab) & 0.51 & Raw water color (lab) \\
\hline 0.79 & Raw water $\mathrm{KMnO}_{4}$ (lab) & 0.50 & $\mathrm{PAC} / \mathrm{KMnO}_{4}$ (on-line) \\
\hline 0.78 & Raw water smell (lab) & 0.50 & Raw water $\mathrm{KMnO}_{4}$ (on-line) \\
\hline 0.76 & Raw water smell, heated (lab) & -0.37 & Raw water $\mathrm{pH}$ (lab) \\
\hline 0.37 & Raw water conductivity (lab) & & \\
\hline \multicolumn{2}{|c|}{ (C) Scaled, sampling moment dataset } & \multicolumn{2}{|c|}{ (D) Interpolated and scaled dataset } \\
\hline Correlation coefficient & Variable & Correlation coefficient & Variable \\
\hline-0.65 & Raw water temperature (on-line) & -0.68 & Raw water temperature (on-line) \\
\hline 0.60 & $\mathrm{PAC} / \mathrm{KMnO}_{4}$ (on-line) & 0.38 & $\mathrm{PAC} / \mathrm{KMnO}_{4}$ (on-line) \\
\hline 0.50 & Raw water color (lab) & 0.37 & Raw water color (lab) \\
\hline 0.49 & Raw water $\mathrm{KMnO}_{4}$ (on-line) & 0.37 & Raw water $\mathrm{KMnO}_{4}$ (on-line) \\
\hline-0.43 & Raw water pH (lab) & 0.26 & Level of Humaljärvi \\
\hline 0.38 & PAC-dose (on-line) & & \\
\hline
\end{tabular}

Table 2. Prediction models and goodness values of the models.

\begin{tabular}{|c|c|c|c|c|}
\hline Model & Variables & RMSE & MAE & $R^{2}$ \\
\hline Model 1 & $\begin{array}{l}\text { - Raw water temperature } \\
\text { - Raw water } \mathrm{KMnO}_{4}(\mathrm{lab}) \\
\text { - Level of surface water } \\
\text { - Raw water coliform bacteria } \\
\text { - WTP returning flow } \\
\text { - Sodium Hypochlorite } \\
\text { - Pressure of filter } 1\end{array}$ & $\begin{array}{l}\text { ANN: } 0.490 \\
\text { MLR: } 0.477\end{array}$ & $\begin{array}{l}\text { ANN: } 0.388 \\
\text { MLR: } 0.377\end{array}$ & $\begin{array}{l}\text { ANN: } 0.244 \\
\text { MLR: } 0.228\end{array}$ \\
\hline Model 2 & $\begin{array}{l}\text { - Raw water temperature } \\
\text { - PAC/KMnO } \\
\text { - Raw water turbidity } \\
\text { - Raw water } \mathrm{KMnO}_{4}\end{array}$ & $\begin{array}{l}\text { ANN: } 0.700 \\
\text { MLR: } 0.561\end{array}$ & $\begin{array}{l}\text { ANN: } 0.532 \\
\text { MLR: } 0.441\end{array}$ & $\begin{array}{l}\text { ANN: } 0.001 \\
\text { MLR: } 0.078\end{array}$ \\
\hline Model 3 & $\begin{array}{l}\text { - Raw water temperature } \\
\text { - Raw water } \mathrm{KMnO}_{4} \\
\text { - Raw water } \mathrm{pH} \\
\text { - PAC-dosage }\end{array}$ & $\begin{array}{l}\text { ANN: } 0.570 \\
\text { MLR: } 0.537\end{array}$ & $\begin{array}{l}\text { ANN: } 0.435 \\
\text { MLR: } 0.427\end{array}$ & $\begin{array}{l}\text { ANN: } 0.088 \\
\text { MLR: } 0.086\end{array}$ \\
\hline Model 4 & $\begin{array}{l}\text { - Raw water temperature } \\
\text { - Raw water } \mathrm{KMnO}_{4} \\
\text { - PAC-dosage }\end{array}$ & $\begin{array}{l}\text { ANN: } 0.544 \\
\text { MLR: } 0.547\end{array}$ & $\begin{array}{l}\text { ANN: } 0.429 \\
\text { MLR: } 0.430\end{array}$ & $\begin{array}{l}\text { ANN: } 0.132 \\
\text { MLR: } 0.071\end{array}$ \\
\hline
\end{tabular}

The amount of residual aluminum is high when raw water is cold even if the raw water $\mathrm{KMnO}_{4}$ and poly-aluminum chloride (PAC) dosage are at the low level. When the raw water temperature is higher and raw water $\mathrm{KMnO}_{4}$ rises the amount of residual aluminum is relatively low. This shows the fact that the effectiveness of the water treatment process is better when raw water is warmer which is very common in most of the WTP. It can be seen that autumn rains and snow melting in the spring affects the quality of raw water and the efficiency of the water treatment process. The raw water color and $\mathrm{KMnO}_{4}$ are at a higher level in the autumn, late winter and spring seasons due to heavy raining and snow melting.

\subsection{Prediction models}

Prediction models were trained using $3 / 4$ of the data and tested using the final $1 / 4$ of the data. Scaled and interpolated dataset was used in modeling and the same variables were used in both MLR and ANN models. Created models and calculated goodness of fit values (RMSE, MAE and $R^{2}$ ) are listed in Table 2. Model 1 was created using variables 

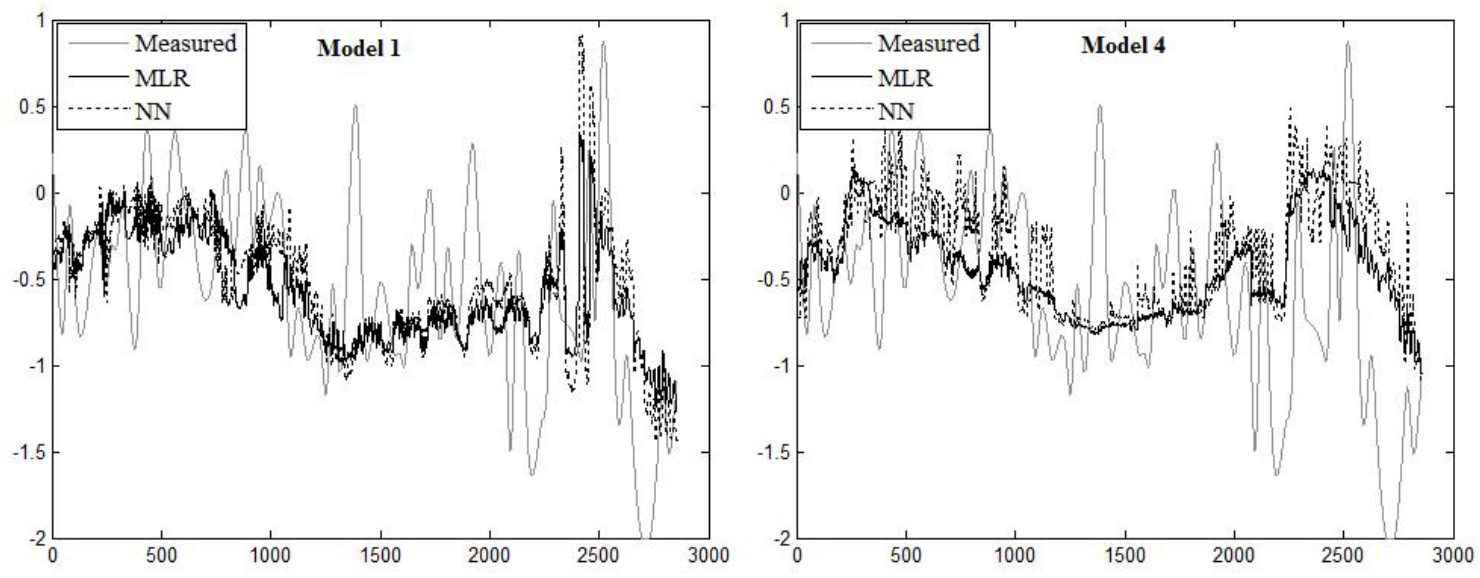

Figure 3. The testing periods of Model 1 (left) and the testing periods of Model 4 (right).

selected by a forward variable selection method. Model 2 was created using the best four variables of variable selection presented in Juntunen et al. (2010) where the best MLP model was achieved with four variables. Manually selected variables were used in Model 3 and Model 4.

During the modeling session it was noticed that there are several different combinations of variables that could be used in modeling the residual aluminum with fairly good accuracy. It was also noticed that the peak values of residual aluminum could be predicted and the accuracy of the model improved only if some laboratory measurements of drinking water were used in the model. This, however, does not give any extra value for real-life application (EWS). The best prediction result was achieved with Model 1 and in Model 2 both ANN and MLR had the lowest accuracy of the models presented in this paper. The difference between ANN and MLR is in Model 2 notably bigger than in Model 1. As it can be seen from Table 2, the error values of the ANN and MLR models were nearly the same in Models 1, 3 and 4, but ANN models seem to be slightly better in every variable set except in Model 2. The accuracy of MLR was better in Model 2 than the accuracy of ANN model. This is an opposite result than presented in Juntunen et al. (2010), where MLP showed a better performance than the MLR method. Modeling methods were quite similar in both studies. Discrepancy between the results could be explained by the significant differences in data pre-processing. In Juntunen et al. (2010) the process data was averaged to 1 day data and combined with daily laboratory data, the measuring period was 275 days, and the dataset was not scaled to range $[-2 \ldots+2]$ using the new nonlinear scaling method.

The results of Model 1 and Model 4 testing periods are shown in Fig. 3 for both ANN and MLR methods. The baseline of the residual aluminum prediction is fairly good with both models. Modeled residual aluminum follows the changes of measured residual aluminum but the peak values could not be predicted. As the calculated results in Table 2 showed, the difference between ANN and MLR models is minor. Model 4 was created using only three variables and the error values were not higher than in Model 1. This is an encouraging result for creating the EWS or on-line control. Almost the same accuracy can be attained with fewer variables.

\section{Conclusions}

The purpose of this work was to analyze the data of the water treatment plant, find out which variables affect the amount of residual aluminum in drinking water, create as simple and reliable prediction models for residual aluminum as possible using ANN and MLR methods and to compare the accuracy of models with each other and to earlier presented results. Same modeling methods and the same data source were used to study the effect of data pre-processing to modeling accuracy. Clear correlations to residual aluminum were found after the dataset was scaled using the new nonlinear scaling method based on generalized norms and skewness. Variables that had the highest correlation to the amount of residual aluminum were among others: the raw water temperature, raw water $\mathrm{KMnO}_{4}$ and $\mathrm{PAC} / \mathrm{KMnO}_{4}$-ratio.

Decent prediction models were created using only a few important variables. The baseline of residual aluminum in drinking water can be predicted with fairly good accuracy with both MLR and ANN models. MLR and ANN methods gave almost the same results. Comparison to earlier results of modeling the residual aluminum at the same water treatment plant was done. In the earlier study the overall modeling accuracy was slightly better. However, in this study the MLR method was found to be better than the ANN method when using the same variables as in the earlier study. Discrepancy between the results can be explained by different kind of data pre-processing. The accuracy of created prediction models could be improved by using different variable selection or using different modeling methods. Yet, the results 
were promising and an early warning system could be created based on these models to give additional information to the process personnel of the water treatment plant.

Acknowledgements. This work was done as a part of the project "Development of a comprehensive water quality management system (POLARIS)". Contribution of relevant persons in Finnsugar Ltd. is greatly acknowledged. The funding of the TEKES (the Finnish Funding Agency for Technology and Innovation) is also acknowledged.

\section{Edited by: L. Rietveld}

\section{References}

Areerachakul, S. and Sanguansintukul, S.: A Comparison between the Multiple Linear Regression Model and Neural Networks for Biochemical Oxygen Demand Estimations, SNLP '09, Eighth International Symposium on Natural Language Processing, 1114, 2009.

Audone, B. and Giunta, G.: Multiple Linear Regression to Detect Shielding Effectiveness Degradations. International Symposium on Electromagnetic Compatibility - EMC EUROPE 2008, 8-12 September 2008, 1-6, 2008.

Baxter, C. W., Stanley, S. J., and Zhang, Q.: Development of a fullscale artificial neural network model for the removal of natural organic matter by enhanced coagulation, J. Water Supply Res. T., 48, 129-136, 1999.

Baxter, C. W., Zhang, Q., Stanley, S. J., Shariff, R., Tupas, R.-R. T., and Stark, H. L.: Drinking water quality and treatment: the use of artificial neural networks, Can. J. Civil Eng., 28 (Suppl. 1), 26-35, 2001.

Beale, M. H., Hagan, M. T., and Demuth, H. B.: Neural Network Toolbox TM 7 User's Guide, Matlab MathWorks Inc., September 2010.

Bowden, G. J., Nixon, J. B., Dandy, G. C., Maier, H. R., and Holmes, M.: Forecasting chlorine residuals in a water distribution system using a general regression neural network, Math. Comput. Model., 44, 469-484, 2006.

Dayhoff, J. E.: Neural Network Architectures: An introduction, Van Nostrand Reinhold, New York, ISBN: 0-442-20744-1, 259 pp., 1990.

Delgrange, N., Cabassud, C., Cabassud, M., Durand-Bourlier, L., and Laine, J. M.: Neural networks for prediction of ultrafiltration transmembrane pressure - application to drinking water production, J. Membrane Sci., 150, 111-123, 1998.

Delgrange-Vincent, N., Cabassud, C., Cabassud, M., DurandBourlier, L., and Laine, J. M.: Neural networks for long term prediction of fouling and backwash efficiency in ultrafiltration for drinking water production, Desalination, 131, 353-362, 2000.

D'Errico, J.: “inpaint_nans-function”, Matlab Central, File Exchance, http://www.mathworks.com/matlabcentral/fileexchange/ 4551 (last access: 26 October 2011), February 2004.

Driscoll, C. and Letterman, R.: Factors regulating residual aluminium concentrations in treated water, Environmetrics, 6, 287309, 1995.

FINLEX: Decree of the Ministry of Social Affairs and Health: Relating to the quality and monitoring of water intended for human consumption, No. 461/2000, Issued in Helsinki on 19 May 2000, 16 pp., http://www.finlex.fi/en/laki/kaannokset/2000/ en20000461.pdf, last access: 26 October 2011, 2000.

George, S., Pandit, P., and Gupta, A. B.: Residual aluminium in water defluoridated using activated alumina adsorption - Modeling and simulation studies, Water Res., 44, 3055-3064, 2010.

Ibarra-Berastegi, G., Elias, A., Arias, R., and Barona, A.: Artificial Neural Networks vs Linear Regression in a Fluid Mechanics and Chemical Modelling Problem: Elimination of Hydrogen Sulphide in a Lab-Scale Biofilter, 2007 IEEE/ACS International Conference on Computer Systems and Applications, 584-587, 2007.

Juntunen, P., Liukkonen, M., Pelo, M., Lehtola, M., and Hiltunen, Y.: Modelling of residual aluminum in water treatment process, in: Proceedings of the 7th EUROSIM Congress on Modelling and Simulation, 6-10 September 2010, Prague, Czech Republic, Vol. 2: Full Papers, 5 pp., 2010.

Juuso, E. K.: Data-based development of dynamic models for biological wastewater treatment in pulp and paper industry, Preprints of the 51st Conference on Simulation and Modelling, 14-15 October 2010, Oulu, Finland, 1-9, 2010.

Juuso, E. K.: Intelligent Trend Indices in Detecting Changes of Operating Conditions, in: Proceedings of UKSim 13th International Conference on Modelling and Simulation - UKSim 2011, Cambridge, UK, 30 March-1 April 2011, 162-167, 2011.

Kulkarni, P. and Chellam, S.: Disinfection by-product formation following chlorination of drinking water: Artificial neural network models and changes in speciation with treatment, Sci. Total Environ., 408, 4202-4210, 2010.

Leakey, H.: Aluminium residual management in drinking water using selection criteria of aluminium based primary coagulants, M.Sc thesis, Royal Roads University Victoria, ISBN: 0-49405113-2, 2004.

Maier, H. R., Morgan, N., and Chow, C. W. K.: Use of artificial neural networks for predicting optimal alum doses and treated water quality parameters, Environ. Modell. Softw., 19, 485-494, 2004.

Matlab Statistics Toolbox ${ }^{\text {TM }} 7$ User's Guide, Matlab MathWorks Inc., April 2011

McLachlan, D. R. C., Bergeron, C., Smith, J. E., Boomer, D., and Rifat, S. L.: Risk for neuropathologically confirmed Alzheimer's disease and residual aluminum in municipal drinking water employing weighted residential histories, Neurology, 46, 401-405, 1996.

Pal, S. K. and Mitra, S.: Multilayer perceptron, fuzzy sets and classification, IEEE Transactions on neural networks, 3, 683-697, 1992.

Shetty, G. R., Malki, H., and Chellam, S.: Predicting contaminant removal during municipal drinking water nanofiltration using artificial neural networks, J. Membrane Sci., 212, 99-112, 2003.

Tranmer, M. and Elliot, M.: Multiple Linear Regression, Catie Marsh Centre for Census and Survey Research, Teaching papers, 47 pp., 2008.

WHO - World Health Organization: Aluminium in Drinking-water: Background document for development of WHO Guidelines for Drinking-water Quality, 2003.

WHO - World Health Organization: Guidelines for drinking-water quality [electronic resource]: incorporating 1st and 2nd addenda, Vol. 1, Recommendations, 3rd Edn., 2008. 